\section{Dramatic presentation of acquired thrombotic thrombocytopenic purpura associated with COVID-19}

The novel SARS-CoV-2 virus causes not only acute respiratory distress, but also diffuse endothelial inflammation. ${ }^{1}$ Acute infections are also well-known triggers for acute thrombotic thrombocytopenic purpura (TTP). ${ }^{2} \mathrm{We}$ herein report the case of an acute TTP episode triggered by a SARS-CoV-2 infection with a dramatic clinical presentation and course. The patient is a 55 -year-old female with a previous episode of TTP caused by bacterial pneumonia 30 years ago, successfully treated at the time with corticosteroids and plasma exchange. A subsequent follow-up was unremarkable, with no TTP relapse. At the end of March 2020, during the COVID-19 epidemic in Italy, she had mild influenza-like symptoms that resolved spontaneously. After 1 month she developed malaise, fatigue, chest discomfort and dyspnea that led to her admission to the emergency room of another hospital in the outskirts of Milan. A chest CT-scan revealed interstitial pneumonia. Two nasopharyngeal swabs for SARSCoV-2 performed at different timepoints (approximately 10 days after the onset of symptoms) were negative but a serological test was positive for specific IgG, featuring a previous exposure to SARS-CoV-2. An empiric treatment with ceftriaxone, azithromycin, hydroxychloroquine and low-dose corticosteroids was started, together with 4,000 IU daily dose of enoxaparin. Low-flow supplemental oxygen ( $2 \mathrm{~L} / \mathrm{min}$ by nasal cannula) was also added. After 3 days there was a rapid drop of the platelet count from $184,000 / \mu \mathrm{L}$ to $14,000 / \mu \mathrm{L}$. Heparin was stopped and, considering her past medical history, a relapse of TTP was suspected. This diagnosis was supported by the concomitant development of anemia (hemoglobin [ $\mathrm{Hb}]$ dropped from 11.4 to $7.4 \mathrm{~g} / \mathrm{dL}$ ), increased hemolysis (lactate dehydrogenase [LDH] increased from 425 to 2,128 $\mathrm{U} / \mathrm{L}$ ) and the presence of schistocytes on the peripheral blood smear. Infusion of fresh frozen plasma was immediately started with no beneficial effect on the hematological parameters and her clinical conditions worsened with the development of severe neurological symptoms (agitation and unconsciousness) and desaturation with the need of high-flow oxygen supplementation $(15 \mathrm{~L} / \mathrm{min}$ with mask and reservoir bag). Her blood lactate levels increased significantly $(15 \mathrm{mmol} / \mathrm{L})$ and at this point the patient was transferred to the intensive care unit of our TTP hub hospital in Milan. On arrival she was unconscious, with respiratory failure and the need of high-flow oxygen and continuous positive airway pressure cycling. Her laboratory tests confirmed intravascular hemolytic anemia (Hb 7.4 g/dL, LDH 18,015 U/L, undetectable haptoglobin) with a negative direct antiglobulin test and thrombocytopenia $(14,000 / \mu \mathrm{L})$ with the presence of numerous schistocytes on the peripheral blood smear and disseminated intravascular coagulation (DIC) with consumption coagulopathy (PT ratio 3.3 , PTT ratio 1.51 , fibrinogen $159 \mathrm{mg} / \mathrm{dL}$, D-dimer 35,187 fibrinogen equivalent units). Plasma ADAMTS13 activity was undetectable together with the presence of a high titer antiADAMTS13 inhibitor $(31 \mathrm{U} / \mathrm{mL})$, confirming the diagnosis of acute immune-mediated TTP associated with severe inflammation and DIC. Laboratory tests were also consistent with multiorgan failure syndrome involving the kidney (creatinine $3.18 \mathrm{mg} / \mathrm{dL}$ ), liver (AST 12602 UI/L, ALT $5625 \mathrm{UI} / \mathrm{L}$, total bilirubin $9.22 \mathrm{mg} / \mathrm{dL}$, direct bilirubin $7.1 \mathrm{mg} / \mathrm{dL}$ ) and the heart ( $\mathrm{T}$ troponin 1,501 $\mathrm{ng} / \mathrm{mL}$, proBNP $61,720 \mathrm{pg} / \mathrm{mL})$. Increased levels of
Table 1. Laboratory parameters at admission and at discharge.

\begin{tabular}{|c|c|c|}
\hline & At admission & At discharge \\
\hline WBC (N) $\left[N^{\circ} / \mu L\right]$ & $40,550(38,420)$ & $8,310(6,390)$ \\
\hline PLT $\left[N^{\circ} / \mu L\right]$ & 14,000 & 207,000 \\
\hline $\mathrm{Hb}[\mathrm{g} / \mathrm{dL}]$ & 7.4 & 11.1 \\
\hline Haptoglobin [mg/dL] & $<10$ & 146 \\
\hline LDH [U/L] & 18,015 & 221 \\
\hline Total bilirubin (indirect) [mg/dL] & $9.22(2.12)$ & 0.55 \\
\hline PT ratio & 3.3 & 0.97 \\
\hline PTT ratio & 1.51 & 0.71 \\
\hline Fibrinogen (mg/dL) & 158 & 172 \\
\hline D-dimer (FEU) & 41,717 & 1,170 \\
\hline FVIII [\%] & ailable pre-caplacizumab & 142 \\
\hline VWF:Ag [\%] & ailable pre-caplacizumab & 221 \\
\hline VWF:Rco [\%] & ailable pre-caplacizumab & 151 \\
\hline ADAMTS13 activity [\%] & $<3$ & 59 \\
\hline anti-ADAMTS13 antibodies [U/mL & 31 & $<12$ \\
\hline CRP $[\mathrm{mg} / \mathrm{dL}]$ & 20.3 & 0.06 \\
\hline Ferritin [ng/mL] & 145,069 & 596 \\
\hline IL-6 [pg/mL] & 6.1 & not available \\
\hline Creatinine [mg/dL] & 3.13 & 0.67 \\
\hline AST [U/L] & 14,081 & 24 \\
\hline ALT [U/L] & 6,516 & 52 \\
\hline $\mathrm{TnT}[\mathrm{ng} / \mathrm{mL}]$ & 1,426 & 30 \\
\hline proBNP $[\mathrm{pg} / \mathrm{mL}]$ & 61,720 & 637 \\
\hline
\end{tabular}

WBC: white blood cell.N, neutrophil.PLT: platelet.Hb, hemoglobin.LDH:lactic dehydrogenase. PT: prothrombin time. PTT,: partial thromboplastin time. FVIII: factor VIII. VWF:Ag, von Willebrand factor antigen.VWF: RCo, von Willebrand factor ristocetin cofactor. ADAMTS13: a disintegrin and metalloproteinase with a thrombospondin type 1 motif member 13. CRP: C-reactive protein. IL-6: interleukin 6. AST: aspartate transaminase. ALT: alanine transaminase. TnT: troponin T. proBNP: prohormone of the brain natriuretic peptide.

C-reactive protein $(20.3 \mathrm{md} / \mathrm{dL})$, procalcitonin $(1.82 \mathrm{ug} / \mathrm{L})$ and IL-6 $(6.1 \mathrm{pg} / \mathrm{mL})$ together with an enormous increase in serum ferritin $(145,069 \mathrm{ng} / \mathrm{mL})$ and neutrophilic leukocytosis $(38,420 / \mu \mathrm{L})$ confirmed the presence of a generalized inflammatory status associated with immune-mediated TTP caused by COVID-19. An extended autoimmune and complement study was performed showing consumed C4 levels, compatible with the acute TTP and COVID-19 infection ${ }^{3,4}$ but excluding an associated autoimmune disease. ${ }^{5}$ Therefore, specific therapy for TTP was promptly started using high dose corticosteroids (methylprednisolone $1 \mathrm{mg} / \mathrm{kg}$ daily) and daily plasma exchange (PEX) (with 1-1.5 plasma volume exchange). Considering the life-threatening presentation, treatment with caplacizumab (10 mg daily after PEX) was also started with the first dose administered before the first PEX. Caplacizumab is a nanobody that binds the A1 domain of the von Willebrand factor (VWF), blocking its interaction with the glycoprotein gpIb-IX-V platelet receptor and therefore preventing platelet aggregation. ${ }^{6}$ Six hours after the first injection of caplacizumab her platelet count rose to $46,000 / \mu \mathrm{L}$ and reached a plateau level of $100,000 / \mu \mathrm{L}$ after 3 days. Due to a persistently altered level of consciousness, a brain CT-scan was performed, showing bilateral frontal parietal petechial hemorrhages and an extended hypodense area involving the right parietal occipital lobe with a smaller image on the left parietal lobe, indicating ischemic lesions. Due to the low platelet 
count, the hemorrhagic cerebral petechiae and the ongoing treatment with caplacizumab, the introduction of antiplatelet therapy and antithrombotic prophylaxis were withheld to avoid intracranial bleeding. When a second brain CT-scan angiography with perfusion study performed after 48 hours confirmed the ischemic nature of the lesions with no increase of the hemorrhagic petechiae, antiplatelet therapy with daily $100 \mathrm{mg}$ aspirin was started. After 9 days of therapy, the basal brain CT-scan showed a complete resolution of the frontal hemorrhagic petechiae and an initial necrotic evolution of the ischemic lesions. At this time, considering her persistent immobilization, antithrombotic prophylaxis with a 4,000 IU daily dose of enoxaparin was initiated. At day 12 the patient had a sudden onset hemoptysis causing acute anemia ( $\mathrm{Hb}$ dropped from 9.0 to $7.7 \mathrm{~g} / \mathrm{dL}$ in a few hours), that required red blood cell transfusion and an urgent chest CT-scan that showed active bleeding from an ectasic artery with a fistulous tract in the main right bronchus. Therefore, caplacizumab, antiplatelet therapy and antithrombotic prophylaxis were stopped, and the patient underwent an emergency transcatheter arterial embolization following treatment with intravenous factor VIII/VWF concentrate (Haemate-P 2,000 IU) to counterbalance the caplacizumab effect on VWF ristocetincofactor activity (VWF:Rco). ${ }^{7}$ The bleeding complication subsided and the patient, after 12 days of therapy with caplacizumab, PEX and steroids obtained a sustained platelet count above $150,000 / \mathrm{uL}$ and the normalization of ADAMTS13 plasma levels (58\%) with antiADAMTS13 antibodies remaining undetectable. Considering the recent major bleeding episode and the fact that ADAMTS13 had become normal, caplacizumab was not restarted. PEX was discontinued after 14 days and the plasma level of ADAMTS13 was monitored every 3 days together with a daily complete blood count.

This clinical case shows an atypical presentation of an acute relapsing TTP during a SARS-CoV-2 infection. We hypothesize that the unusual dramatic presentation of this case is explained by her recent SARS-CoV-2 infection that, by causing a severe systemic inflammation, led to diffuse activation of the microvascular endothelium that triggered the acute TTP onset. The low ADAMTS13 levels contributed to the endothelial and coagulation activation with overt $\mathrm{DIC}^{8}$ leading to multiple organ ischemia and related failure. The diffuse involvement of the microvascular wall may also explain the bleeding tendency. ${ }^{9}$ The therapeutic choices in this patient were challenging considering the complex picture of microthrombosis in different organs, ischemic stroke and lung bleeding all together. Caplacizumab played a crucial role at the onset of the disease, increasing quickly the platelet count. However, the addition of an antiplatelet and anticoagulant therapy led to a markedly unbalanced hemostasis, resulting in a life-threatening acute bleeding episode despite a normal platelet count. Another atypical aspect of this case is the sluggish platelet count norma- lization above $150,000 / \mu \mathrm{L}$ (only after 12 days of treatment), ${ }^{6}$ despite the active effect of caplacizumab demonstrated by the suppressed activity of the VWF (VWF:RCo $<6 \%$ ). This unusually long duration of treatment to obtain a platelet count normalization may be attributed to the severe inflammatory state secondary to systemic endothelial activation that, once subsided, led to the patient discharge after 31 days from admission, with a normal platelet count and normal ADAMTS13 levels.

\section{Marco Capecchi, ${ }^{1}$ Cristina Mocellin, ${ }^{2}$ Chiara Abbruzzese, ${ }^{3}$} Ilaria Mancini, ${ }^{4}$ Daniele Prati ${ }^{2}$ and Flora Peyvandi, ${ }^{4}$

${ }^{1}$ Università degli Studi di Milano, Department of Biomedical Sciences for Health; ${ }^{2}$ Fondazione IRCCS Ca' Granda Ospedale Maggiore Policlinico, Department of Transfusion Medicine and Haematology; ${ }^{3}$ Fondazione IRCCS Ca' Granda Ospedale Maggiore Policlinico, Department of Anesthesia and Intensive Care; ${ }^{4}$ Università degli Studi di Milano, Department of Pathophysiology and Transplantation and 'Fondazione IRCCS Ca' Granda Ospedale Maggiore Policlinico, Angelo Bianchi Bonomi Hemophilia and

Thrombosis Center, Milan, Italy

Funding: this work was supported by the Italian Ministry of Health, Bando Ricerca Corrente.

Correspondence: FLORA PEYVANDI - flora.peyvandi@unimi.it doi:10.3324/haematol.2020.262345

\section{References}

1. Zsuzsanna V, Andreas JF, Peter S, et al. Endothelial cell infection and endotheliitis in COVID-19. Lancet. 2020;395(10234):1417-1418.

2. Saha M, McDaniel JK, Zheng XL. Thrombotic thrombocytopenic purpura: pathogenesis, diagnosis and potential novel therapeutics. J Thromb Haemost. 2017;15(10):1889-1900.

3. Réti M, Farkas P, Csuka D, et al. Complement activation in thrombotic thrombocytopenic purpura. J Thromb Haemost. 2012; 10(5):791-798.

4. Cugno M, Meroni PL, Gualtierotti R, et al. Complement activation in patients with COVID-19: a novel therapeutic target. J Allergy Clin Immunol. 2020;146(1):215-217.

5. Bérangère SJ, Coppo P, Veyradier A. Thrombotic thrombocytopenic purpura. Blood. 2017;129(21):2836-2846.

6. Peyvandi F, Scully M, Kremer Hovinga JA, et al. Caplacizumab for acquired thrombotic thrombocytopenic purpura. N Engl J Med. 2016; 374(6):511-522.

7. Scully M, Cataland SR, Peyvandi F, et al. Caplacizumab treatment for acquired thrombotic thrombocytopenic purpura. N Engl J Med. 2019; 380(4):335-346.

8. Hyun J, Kim HK, Kim JE, et al. Correlation between plasma activity of ADAMTS-13 and coagulopathy, and prognosis in disseminated intravascular coagulation. Thromb Res. 2009;124(1):75-79.

9. Nasser M, Cottin V. Alveolar hemorrhage in vasculitis (primary and secondary). Semin Respir Crit Care Med. 2018;39(4):482-493. 